\title{
A New DFT-HQ Channel Estimation Algorithm in OFDM Systems for Sparse Multipath Channels
}

\author{
Chen $\mathrm{Li}^{(1)} \quad$ Liu Yuan-an $^{(1)}$ Gao Jin-chun ${ }^{(1)} \quad$ Xie Gang ${ }^{(2)}$
}

(1)(Beijing Key Laboratory of Work Safety Intelligent Monitoring, Beijing University of Posts and Telecommun ications, Beijing 100876, China, Email:sczylzjscl@ 163.com)

\author{
(2)(Beijing Key Laboratory of Network System Architecture and Convergence, Beijing University of Posts and \\ Telecommunications, Beijing 100876, China, Email:xiegang@bupt.edu.cn)
}

\begin{abstract}
In this paper, we propose an improved discrete Fourier transform (DFT)-based channel estimation scheme for Orthogonal Frequency Division Multiplexing (OFDM) systems. The proposed algorithm uses Hannan-Quinn (HQ) information criterion to estimate the number of most significant paths and the multipath time delays. With these channel parameters, Our scheme can reduce the noise impact and improve the performance of traditional DFT channel estimation scheme. Compared with other information criterion, such as the minimum description length (MDL) and the generalized Akaike information criterion (GAIC) criterion, the proposed scheme is found to have better performance.
\end{abstract}

Keywords-sparse channel estimation; discrete Fourier transform (DFT); Hannan-Quinn (HQ) criterion; OFDM;

\section{INTRODUCTION}

OFDM has the most promise as a future high data rate wireless communication system due to its high bit-rate transmissions over frequency selective fading channels. However, the high-rate and spectrum efficient OFDM systems generally require estimation and tracking of the fading channel parameters to perform coherent demodulation. Channel estimation is important in OFDM systems.

Channel estimation methods mainly include blind channel estimation and pilot-based channel estimation. The blind channel estimation needs a large amount of data and the convergence rate is very slow. We usually use the pilot-based channel estimation methods because it has better performance. In this paper, only the pilot-based channel estimation methods are investigated.

Depending on the processing domain, pilot-based channel estimation of OFDM systems can be classified into two types: Frequency domain methods and Time domain methods. The least square (LS) estimation is the simplest frequency domain channel estimation method that does not use any information about the channel. Its performance may badly damaged by the noise. The minimum mean square error (MMSE) estimation can achieve better performance by using channel statistics information such as channel autocorrelation matrix and noise variance. Moreover, MMSE methods requires very large amount of computation, such as matrix inversion. In order to reduce the complexity of the MMSE, singular value decomposition has been used in [1][2]. However, these methods still need exact channel autocorrelation matrix which is hard to require in practice.

The time domain channel estimation method focuses on the time domain by using the discrete Fourier transform (DFT) processing. We can modify the LS and the MMSE using the fact that channel impulse response (CIR) is not longer than the guard interval in OFDM systems. However, the DFT estimation cannot eliminate the noise inside the guard interval. [3][4] proposed threshold-based DFT channel estimation methods. But the performance is bad in low SNR. Since the channel energy is usually concentrated in limited significant paths, some people tried to estimate the significant paths and path delays. In [5], Yang proposed a channel estimation method using minimum description length (MDL) .Yang's method needs eigenvalue decomposition and the computational complexity is high. After Yang, Raghavendra proposed a parametric channel estimation scheme using the generalized Akaike information criterion (GAIC)[6]. This method uses LS scheme and GAIC to estimate the channel length and tap positions and can effectively reduces the signal space of LS estimator, and hence improves the performance effectively.

In this paper, we propose a DFT-HQ channel estimation scheme using the Hannan-Quinn(HQ) criterion. This scheme not only has a better performance.

In this paper, (. $)^{T}$ denotes transposition; (. $)^{H}$ denotes Hermitian transposition, and $I_{N}$ is the $N \times N$ identity matrix.

\section{SYSTEM MODEL AND PROBLEM FORMULATION}

\section{A. Channel model}

The sparse multipath channel can be described as

$$
h(t, \tau)=\sum_{l=0}^{L-1} h_{l}(t) \delta\left(\tau-\tau_{l}\right)=\sum_{l=0}^{L-1} h_{l}(t) \delta\left(\tau-\lambda_{l} T_{s}\right)
$$

where $L$ denotes the number of significant channel paths, $\tau_{l}=\lambda_{l} T_{s}$ is the time delay of the $l$ th significant channel path, $T_{s}$ is the sampling interval of the receiver. In the above model, we assume that all channel delay fall at the sampling instants of the receiver and there is no channel power loss caused by sampling time mis match, namely, 
$\lambda_{l}$ is an integer. We also assume that different channel paths are independent, and is a wide stationary Gaussian process whose mean value function is zero and variance function is $\sigma_{l}^{2}$ with $\sum_{l=0}^{L-1} \sigma_{l}^{2}=1$.Because the channel is sparse, the maximum time delay $\lambda_{L-1}>L$.

\section{B. System model}

Consider an OFDM system that consists of $N$ subcarriers and $M$ OFDM symbols in each frame. The OFDM transmitter uses an inverse DFT (IDFT) of size $N$ for modulation. The last $L_{c p}$ samples of IDFT output are appended as cyclic prefix to form one OFDM symbol of $N+L_{c p}$ samples. Then it passes through a multipath channel. At the receiver, the $\mathrm{CP}$ is removed and an $N$ point DFT is performed on each OFDM symbol. The received vector can be expressed as

$$
\mathbf{Y}=\mathbf{X H}+\mathbf{W}=\mathbf{X F h}+\mathbf{W}
$$

Where $\mathbf{Y}=\left[Y_{1}, Y_{2}, \ldots, Y_{N}\right]$ is the received vector, $X$ is a diagonal matrix of transmitted data, $\mathbf{X}=\operatorname{diag}\left[X_{1}, X_{2}, \ldots, X_{N}\right]$ and $\mathbf{H}$ is the DFT of the time domain channel response $\mathbf{h}, \mathbf{F}$ is the $N \times N$ DFT matrix, $\mathbf{W}$ is a zero-mean complex Gaussian noise which has an auto-correlation matrix $\sigma_{n}^{2} \mathbf{I}_{\mathbf{N}}, \sigma_{n}^{2}$ is the noise variance.

\section{Traditional DFT channel estimation}

DFT-based channel estimation is a good scheme for OFDM system with the tradeoff between complexity and performance. In DFT scheme, we first use LS estimator in frequency domain and then transform the results to time domain. Since the channel energy is usually concentrated in limited significant paths and others are noise, so only the limited paths are reserved and the rest is set to zero. We can know this point from Fig.1, the channel has 6 significant paths. In our paper, we use the comb pilots. In each OFDM symbol, $P=N / D$ pilots are transmitted, where $D$ is the frequency interval between two adjacent pilots. Considering the pilot design criterion, $P$ should be larger than $\lambda_{L-1}$. The traditional DFT channel estimation algorithm has 4 steps shown as below.

Step1: After the OFDM demodulation, we first use the LS estimation to obtain channel estimation on pilot subcarriers.

$$
H_{L S}(k)=Y(k) / X(k)=H(k)+W(k) / X(k)
$$

Where $k \in P S, P S$ is the set of pilot subcarrier.

Step2: Use the IDFT to transform the frequency response to time domain

$$
h_{l s}(n)=\operatorname{IDFT}\left[H_{L S}(k)\right]=h(n)+\hat{w}(n)
$$

Where $\hat{w}(n)=\operatorname{IDFT}[W(k) / X(k)], \quad n=1,2, \ldots P, P$ is the number of pilots for each OFDM symbol.

Step3: In order to restrain the effect of the noise, the channel impulse response (CIR) are set to zeros when $n \geq L_{c p}$

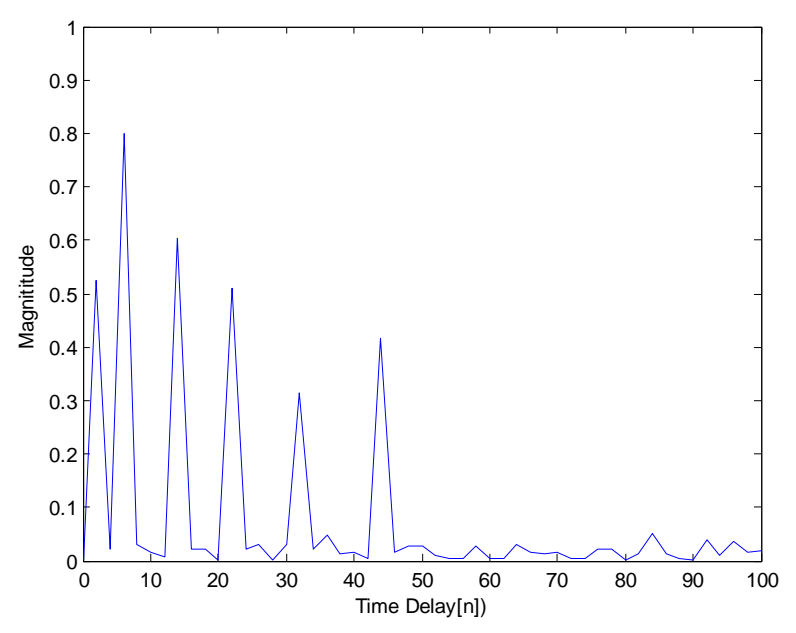

Fig. 1 channel impulse response in time domain

$\hat{h}_{D F T}(n)=\left\{\begin{array}{lr}h_{l s}(n) & 0 \leq n \leq L_{c p} \\ 0 & n \geq L_{c p}\end{array}\right.$

Step4: Transform the time domain response back to frequency domain to obtain the frequency response on all subcarrier.

$\hat{H}_{D F T}(k)=D F T\left[\hat{h}_{D F T}(n) 0 \ldots 0\right]$

Where $k=1,2, \ldots, N$.

In fact, the number of significant channel paths $L$ is smaller than $L_{c p}$.From the figure 1, we can see that knowledge of the number of significant channel paths and the multipath time delays will help in improving the performance of the DFT estimator. In this paper, we use the HQ criterion to estimate these channel parameters. This will be discussed in the next section.

\section{PROPOSED DFT-HQ CHANNEL ESTIMATION}

A channel is said to be sparse if most of its power concentrated on several paths. We need estimate the significant channel paths and multipath time delays. In fact, a similar problem has already been widely investigated in detection of the number of the signal sources. Now we use the HQ criterion to detect the number of channel paths.

Due to the sparsity of the multipath channel, We can construct a $P \times 1$ vector $\mathbf{h}_{\mathbf{m}}=\left[h_{0, m}, h_{1, m}, \ldots, h_{P-1, m}\right]$, where $m$ means the OFDM symbol.

$$
h_{i, m}= \begin{cases}h_{l, m} & i=\lambda_{l}, l=0,1, \ldots, L-1 \\ 0 & i \neq \lambda_{l}, l=0,1, \ldots, L-1\end{cases}
$$

Consider

$$
\begin{aligned}
\mathbf{R} & =E\left\{\mathbf{H}_{L S} \mathbf{H}_{L S}{ }^{\mathbf{H}}\right\} \\
& =E\left\{\mathbf{H H}^{\mathbf{H}}\right\}+E\left\{\mathbf{W} \mathbf{W}^{\mathbf{H}}\right\} \\
\mathbf{H} & =\mathbf{G h}
\end{aligned}
$$

where $\mathbf{G}$ is the $P \times P$ DFT matrix.

$$
E\left\{\mathbf{H H}^{\mathbf{H}}\right\}=\mathbf{G} \tilde{\boldsymbol{\psi}} \mathbf{G}^{\mathbf{H}}
$$


$\tilde{\boldsymbol{\psi}}=E\left\{\mathbf{h}_{\mathbf{m}} \mathbf{h}_{\mathbf{m}}^{\mathbf{H}}\right\}$ is a $P \times P$ diagonal matrix whose ith diagonal element is $\tilde{\psi}_{i}$

$$
\tilde{\psi}_{i}=\left\{\begin{array}{lll}
\sigma_{l}^{2} & i=\lambda_{l}, & l=0,1, \ldots L-1 \\
0 & i \neq \lambda_{l}, & l=0,1, \ldots L-1
\end{array}\right.
$$

We can see that the number of channel paths is the number of non-ze ro diagonal elements of $\tilde{\boldsymbol{\psi}}$.Actually, $\tilde{\boldsymbol{\psi}}$ is hard to be accurately obtained. We can estimate the $R$.The diagonal elements of $R$ can also reflect the number of channel paths. For an OFDM frame composed of $M$ OFDM symbols, $\mathbf{R}$ can only be approximately obtained by

$$
\mathbf{R} \approx \frac{1}{M} \sum_{0}^{M-1} \mathbf{H}_{L S} \mathbf{H}_{L S}^{\mathbf{H}}
$$

From the above equation, it cannot guarantee that $R$ is a diagonal matrix. However, the $\left\{\lambda_{l} \mid l=0,1, \ldots L-1\right\}$ th diagonal elements of $R$ are generally larger than the other diagonal elements especially when the SNR is high. So the diagonal elements of $R$ can still be utilized to estimate the channel parameters.

Our algorithm can be described as below. First, we use the HQ criterion to estimate the number of significant paths and time delays. Second, we use these channel parameters to improve the DFT channel estimation.

1. Calculate $\mathbf{R}$ and extract its diagonal elements, and denote them as $\theta=\left[\theta_{1}, \theta_{2}, \ldots, \theta_{p-1}\right]^{T}$.

2. Sort the $\theta$ in descending order $\bar{\theta}=\left[\bar{\theta}_{0}, \bar{\theta}_{2}, \ldots, \bar{\theta}_{p-1}\right]^{T}$.

3. By HQ criterion, the number of channel paths can be estimated as

$$
L=\arg \min _{l=\{0,1 \ldots P-1\}}\left(f_{1}(l)-f_{2}(l)\right) K+f_{3}(l)
$$

Where

$$
\begin{gathered}
f_{1}(l)=(P-l) \ln \left(\frac{\sum_{i=l}^{p-1} \bar{\theta}_{i}}{p-l}\right) \\
f_{2}(l)=\sum_{i=l}^{p-1} \ln \bar{\theta}_{i} \\
f_{3}(l)=\frac{1}{2} l(2 P-l) \ln \ln K
\end{gathered}
$$

4. After $L$ is estimated, find the indices of the $L$ largest values of $R$. The estimated time delays are the index values.

With these estimated channel parameters, the channel estimation are obtained as

$$
\hat{h}_{D F T-H Q}(n)=\left\{\begin{array}{lr}
h_{l s}(n) & n \in\left\{\lambda_{l} \mid l=0,1, \ldots L-1\right\} \\
0 & n \notin\left\{\lambda_{l} \mid l=0,1, \ldots L-1\right\}
\end{array}\right.
$$

Transform the time domain response back to frequency domain to obtain the frequency response on all subcarrier.

$$
\hat{H}_{D F T-H Q}(k)=D F T\left[\hat{h}_{D F T-H Q}(n) 0 \ldots .0\right]
$$

Where $k=1,2, \ldots, N$

\section{SimULATION RESULTS}

In this section, a 6-path channel with time delays $[0,0.2,0.4,1.5,2.4,5]$ us .For the OFDM systems, $N=512$ subcarriers,$P=64$ pilots and the number of CP samples is 64.

We compare the proposed algorithm and other algorith ms using two metrics, the correct estimation of all channel parameters probability (CECP) and MSE.

$\mathrm{CECP}=\operatorname{Pr}\{$ Correct estimation of channel parameters $\}$

However, it is not easy to get a closed-form expression for the CECP, and its value can only be obtained through simu lation.

$\mathrm{CECP}=($ Number of simulation runs where all channel parameters are correctly estimated)/(total number of simulation runs)

$$
\mathrm{MSE}=E\left\{|H[k]-\hat{H}[k]|^{2}\right\}
$$

In the figures below, the performance of MDL and GA IC criterion is given to evaluate the proposed scheme.

\section{A. Performance vs. Frame Lengths}

From the equation (12), we can see the frame length $\mathrm{K}$ affect the accuracy of the matrix $\mathbf{R}$. In this subsection, we set $\mathrm{SNR}=30 \mathrm{~dB}$, we show the performance variation for varying frame length $K$.

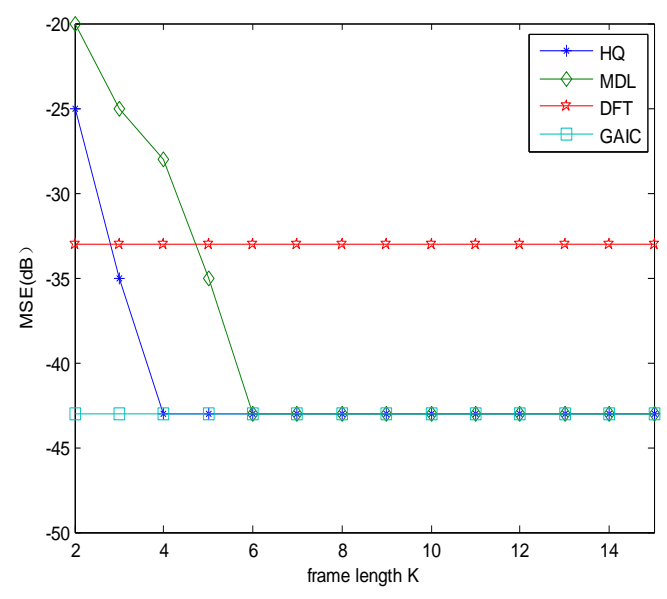

Fig. 2 MSE performance comparison versus frame lengths

From Fig.2, we can see that DFT and GAIC estimation schemes don't change versus the frame length $\mathrm{K}$ because they needn't compute the correlation matrix. The GAIC performs better than the DFT. The proposed HQ estimation scheme performs better than the MDL when the number of $K$ is small. After $K$ becomes lager than 6, these two methods almost have the same performance.

\section{B. Performance vs.SNR}

In this subsection, we give the performance of the proposed scheme and the others versus the SNR when the frame length $K$ is 200 . 


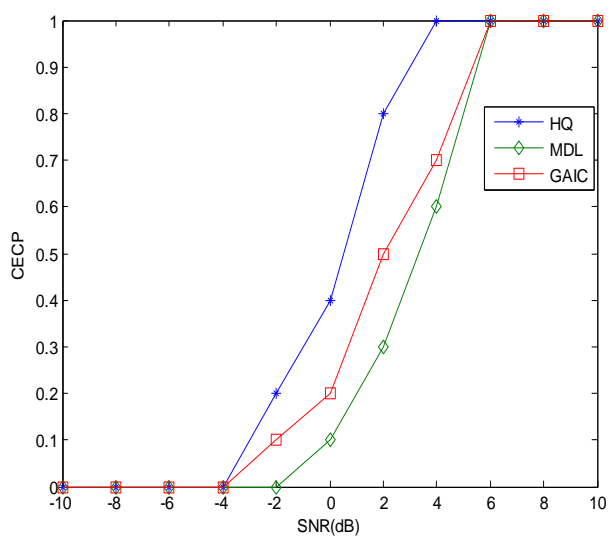

Fig. 3 CECP performance comparison versus SNR

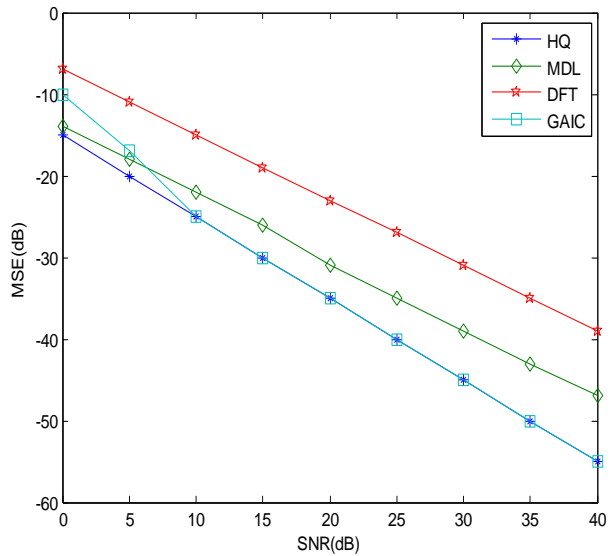

Fig. 4 MSE performance comparison versus SNR

Figure 3 show that all parametric channel estimation schemes can correctly the channel parameters when the SNR is high. However, the channel parameters cannot be correctly estimated in the low SNR area because channel path power is overwhelmed by the noise. Our proposed scheme performs better than these two parameter estimation scheme. For the MSE, in figure 4, we can observe that our scheme is best among these methods in low SNR area. In high SNR area, we can see that our scheme and GAIC perform almost the same and better than others. The performance of DFT scheme can be raised substantially after we use the HQ criterion.

\section{CONCLUSIONS}

In this paper, we have presented a new channel estimation scheme for sparse multipath channels. This scheme uses the HQ criterion to estimate the number of significant multipath and time delays, and then uses these parameters to improve the performance of channel estimation. From the simulation results, we can see our scheme performs better than the other traditional channel estimation schemes.

\section{ACKNOWLEDGMENT}

This work was supported in part by Important National Science and Technology Specific Projects (No. 2012ZX03003001-004), Canada-China Scientific and Technological Cooperation (No. 2010DFA11320), Project 61272518 and 61170275 supported by the National Natural Science Foundation of China (NSFC).

\section{REFERENCES}

[1] O. Edfors, M. Sandell, et al. "OFDM channel estimation by singular value decomposition," IEEETran. Commun., 46:931-939, 1998.

[2] M. Noh, Y. Lee, and H. Park, "A low complexity LMMSE channel estimation for OFDM",IEE Proc. Commun., 2006, 153, (5), pp. 645-650

[3] Y. Kang,K. Kim, H. Park, "Efficient DFT-based channel estimation for OFDM systems on multipath channels," IEEE Commun. IET., vol.1, pp. 197-202, April. 2007.

[4] Y.-S. Lee et al. Channel estimation based on a time-domain threshold for OFDM systems. IEEE Tran. Broadcast., 55(3):656662, 2009.

[5] B. Yang, K. B. Letaief, R. S. Clieng, and Z. Cao, "Channel estimation for OFDM transmission in multipath fading channels based on parametric channel modeling," IEEE Trans. Commun., vol. 49, pp. 467-478, Mar.2001

[6] M. R. Raghavendra and K. Giridhar,“ Improving channel estimation in OFDM systems for sparse multipath channels,"IEEE Signal Processing Lett., vol. 12, pp. 52-55, Jan. 2005.

[7] E. J. Hannan and B. G. Quinn, "The determination of the order of an autoregression,"J. Roy. Stat. Soc., vol. 41, pp. 190-195, 1979.

[8] C. Yeh and Y. Lin. Channel estimation using pilot tones in OFDM systems. IEEE Tran. Broadcast., 45(4):400-409, 1999.

[9] Jeong-Wook Seo, Jung-Wook Wee, Yong-Suk Park, Jong-Ho Paik, and Won-Gi Jeon," DFT-based channel estimation using CIR adaptation in OFDM systems," IEEE Advanced Communication Technology Conf., pp. 23-26, Feb. 2007.

[10] G. Auer, S. Sand, and A. Dammann, "Comparison of low complexity OFDM channel estimation techniques," inProc. 8th International OFDM Workshop, Hamburg, Germany, pp. 157-161, Sept. 2003.

[11] S. Y. Liu, F. F. Wang, R. R. Zhang, Y. A. Liu, "A Simplified Parametric Channel Estimation Scheme for OFDM Systems," IEEE Transactions on Wireless Communications, vol. 7, no. 12, pp. 5082 - 5090, Dec. 2008. 